\title{
Parkinson's disease patients' perspective on context aware wearable technology for auditive assistance
}

\author{
Marc Bächlin ${ }^{\dagger *}$, Meir Plotnik ${ }^{\ddagger}$, Daniel Roggen ${ }^{\dagger}$, Noit Inbar ${ }^{\ddagger}$, Nir Giladi ${ }^{\ddagger}$, Jeffrey Hausdorff ${ }^{\ddagger}$, Gerhard Tröster ${ }^{\dagger}$ \\ †Wearable Computing Lab, Swiss Federal Institute of Technology Zürich, Switzerland \\ ${ }^{\ddagger}$ Laboratory for Gait and Neurodynamcis, Tel Aviv Sourasky Medical Center, Israel \\ ${ }^{*}$ Corresponding author: baechlin@ife.ee.ethz.ch
}

\begin{abstract}
In this paper we present a wearable assistive technology for the freezing of gait (FOG) symptom in patients with Parkinson's disease (PD), with emphasis on subjective user appreciation. Patients with advanced PD often suffer from FOG, which is a sudden and transient inability to move. It often causes falls, interferes with daily activities and significantly impairs quality of life. Because gait deficits in PD patients are often resistant to pharmacologic treatment, effective nonpharmacologic treatments are of special interest.

We have developed an ambulatory device that detects FOG episodes in real-time and provides an automatic cueing sound until the subject resumes walking. We tested our device on ten PD patients. Eight patients experienced FOG during our study. Over $8 \mathrm{~h}$ of data has been recorded and 237 FOG events have been identified by professional physiotherapists in a post-hoc video analysis. The device detected the FOG events with a sensitivity of $73.1 \%$ and a specificity of $81.6 \%$ on a $0.5 \mathrm{sec}$ frame based evaluation.

The most important finding of the study is that we can provide online assistive feedback for FOG events in PD patients. Based on subjective reports, the majority of patients indicated that the context aware automatic cueing is beneficial for them.
\end{abstract}

\section{INTRODUCTION}

\section{A. Parkinson's disease}

Parkinson's disease (PD) is a common neurological disorder caused by a progressive loss of dopaminergic and other subcortical neurons [1]. PD often includes impaired motor skills as part of its symptoms [2]. Beside a flexed posture, tremor at rest, rigidity, akinesia (or bradykinesia) and postural instability, motor blocks, i.e., complete inability to move, are a common negative effect of PD.

Clinical assessment of PD is largely based on subjective patient reports. The Hoehn and Yahr (H\&Y) scale is a commonly used system for describing the symptoms of PD progress. The scale allocates stages from $I$ to $V$, to indicate the relative level of disability [3]. The five stages are:

I: Symptoms on one side of the body only.

II: Symptoms on both sides of the body. No impairment of balance.

III: Balance impairment. Mild to moderate disease. Physically independent.

IV: Severe disability, but still able to walk or stand unassisted.

V: Wheelchair-bound or bedridden unless assisted.

\section{B. Freezing of gait}

About $50 \%$ of patients in the advanced stages of the PD are affected by the freezing of gait (FOG) symptom [4]. Patients, who frequently experience FOG report that during the freezing episode their feet are inexplicably glued to the ground [5].

Schaafsma et al. have divided FOG into start hesitation, turn hesitation, hesitation in tight quarters, destination hesitation and open space hesitation [5].

A survey among the 12000 members of the German Parkinson Association, which was answered by 6620 patients, has shown that $47 \%$ of the patients experience FOG. FOG occurs more frequently in men than in women and less frequently in patients whose main symptom is tremor [6]. $10 \%$ of respondents with mild PD symptoms and $80 \%$ of those severely affected regularly experience freezing.

FOG is associated with substantial social and clinical consequences for patients. In particular, it commonly causes falls [7], interferes with daily activities, and significantly impairs quality of life [8].

\section{Limits of pharmacological treatment of FOG}

Most common form of treatment to manage the motor symptoms of PD is Levodopa (LD). LD is the metabolic precursor to dopamine and is used to replace endogenous dopamine at the striatum. However, only $1-5 \%$ of LD enters the dopaminergic neurons. The remaining $L D$ is often metabolised to dopamine elsewhere, causing a wide variety of side effects.

The medication cycle between two consecutive intakes is roughly divided to two periods, the $O N$ period in which the LD is effective, and the $O F F$ period in which the influence of the medicine has subsided. By determining dosage and frequency of daily LD consumption, the clinicians attempt to maximize the $O N$ periods and to minimize the $O F F$ period. As the disease advances more frequent $L D$ administration is necessary [9]. In addition, the development of involuntary movements and the $O N / O F F$ phenomenon (motor response fluctuations un-correlated with the expectation from the daily medications intake schedule) can limit mobility and complicate dosing.

Gait deficits and FOG are often resistant to pharmacologic treatment [7]. Especially when FOG occurs during the $O N$ period, it does not respond to dopaminergic therapy. Therefore, effective non-pharmacologic treatments need to be developed as an adjunct therapy to relieve symptoms and improve mobility. 


\section{State of the art in non-pharmaceutical treatment of FOG}

In terms of non-pharmaceutical treatment, previous work has shown that gait performance in PD can be improved by applying continuous external rhythmic auditory, visual or somatosensory cues [10]-[12].

An extensive review study by Lim et al. on articles published from 1966 to January 2005 on external rhythmical cueing to support PD patients (159 screened studies) has shown best-evidence for improving walking speed with the help of auditory cues [12]. Insufficient evidence was found for the effectiveness of visual and somatosensory cueing. However, it is unclear whether positive effects identified in the laboratory can be generalized to improved activities of daily living (ADLs) and reduced frequency of falls in the community. In addition, the sustainability of a cueing training program remains uncertain [12].

Nieuwboer et al. evaluated the effect of cueing training in PD patients at home. 153 patients with PD have taken part in the 3-week home cueing program, with a total of nine treatment sessions of $30 \mathrm{~min}$ each. They showed that cueing training at home has specific effects on gait, freezing and balance. The decline in effectiveness of intervention effects underscores the need for permanent cueing devices and followup treatment [13].

The main limitation of the external cueing approach used so far is the continuous nature of the intervention. For example, it would be most inconvenient for a patient to move around with constant ticking sound in daily life situations. In order to overcome this limitation, our objective is to develop and evaluate a system, that provides cueing only in the context of impaired performance. In this study we focused on the actual FOG episodes.

\section{E. Paper Contribution}

In the present study we address the shortcoming of the state of the art, which is the continuous cueing that becomes inconvenient in real-world daily life. In detail the contributions are:

1) Testing and evaluating a wearable system together with algorithms to detect FOG online and provide automatically auditive cueing for the PD patients;

2) Evaluating PD patients' and physiotherapists' perceptions of the technology with the help of questionnaires.

\section{WEARABLE DEVICE FOR ONLINE FOG DETECTION AND AUDITORY CUEING}

\section{A. Hardware}

Figure 1 shows a patient wearing the device we studied. Two accelerometer sensors are attached to the shank (just above the ankle) and the thigh (just above the knee) using an elasticized strap and Velcro. A third sensor is attached to the belt of the patient. Each acceleration sensor, including a rechargeable Li-ion battery, are $27 \times 47 \times 12 \mathrm{~mm}^{3}$ in size and weights $\approx 22$ grams [14].

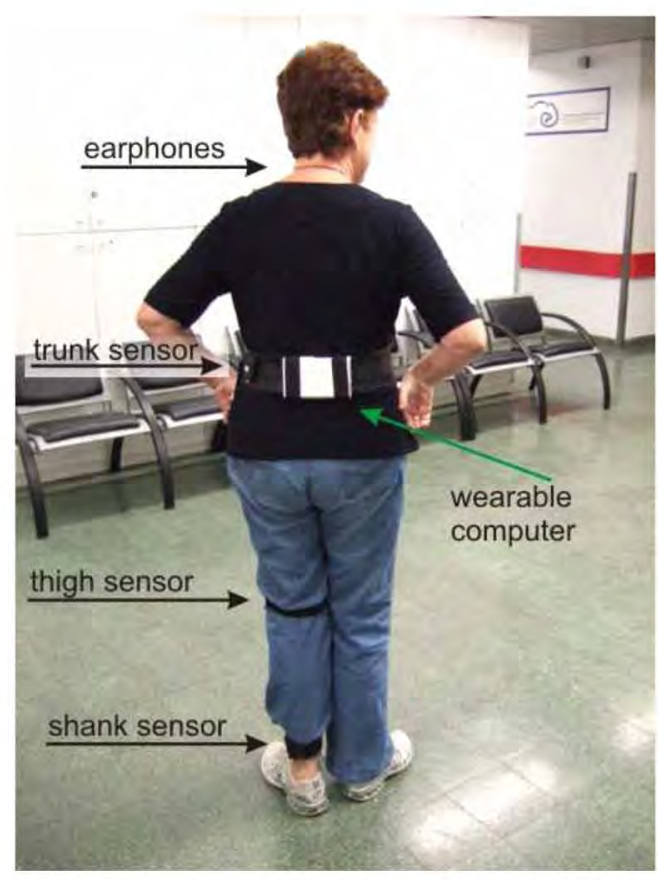

Fig. 1. FOG detection and feedback device worn by a patient. Sensors are attached to the shank (just above the ankle) and the thigh (just above the knee) using an elasticized strap and Velcro. A third sensor is attached to the belt where also the wearable computer is attached to.

The wearable computer $\left(132 \times 82 \times 30 \mathrm{~mm}^{3}, 231\right.$ grams $)$ is also attached to the belt, placed around the trunk. The acceleration data $(64 \mathrm{~Hz})$ are transmitted over a wireless Bluetooth link to the computing system for real-time identification of FOG. Earphones placed around the subject's neck and connected to the computing system produce a $1 \mathrm{~Hz}$ ticking sound whenever an FOG episode is identified and last until the subject resume walking.

\section{B. Online FOG detection algorithm}

A phenomenological study of Schaafsma et al. using video analysis has shown that FOG is often associated with a 'trembling' of the legs in an effort to overcome the block [5]. Although the underlying pathology of FOG is still unknown, Schaafsma speculated that FOG is an atypical form of action legs dystonia or dystonic tremor [15].

A laboratory study of insole pressure demonstrated an increase in high-frequency (2-6 Hz) components during freezing, that may represent an attempt to overcome the block or the output of multiple pathologic neural networks, that interfere with the normal locomotor pattern [16].

Moore et al. analyzed the power spectra of the vertical linear acceleration of the left shank of 11 PD patients [17]. They found out that high-frequency components of leg movement during FOG in the 3-8 $\mathrm{Hz}$ band were not apparent during volitional standing or during walking. For an objective method to identify FOG offline, Moore introduced a freeze index (FI) defined as the power in the 'freeze' band divided by the power in the 'locomotor' band $(0.5-3 \mathrm{~Hz})$. FOG can be detected 

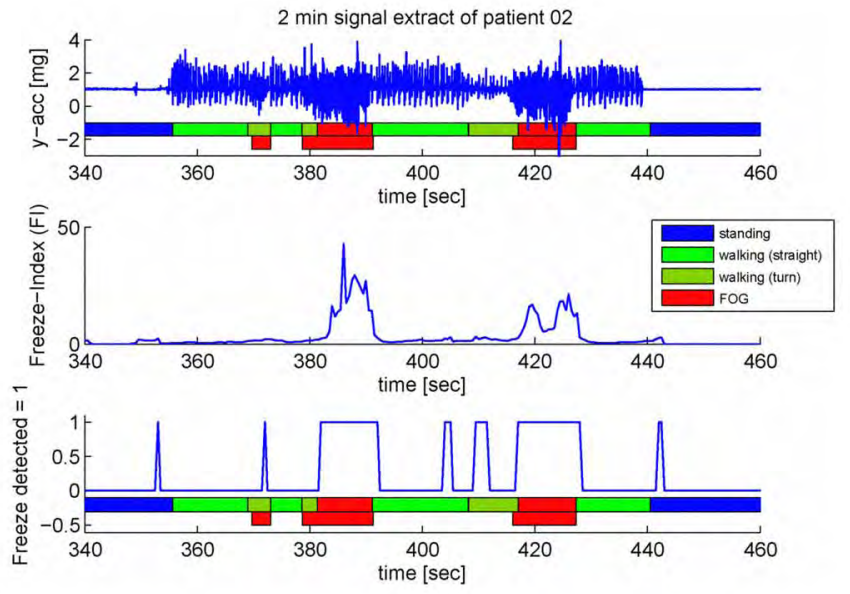

Fig. 2. A 2 min signal extract from a PD patient (patient 02) demonstrates the FOG detection algorithm. Top: The raw vertical acceleration of the shank during the study with the real-time annotation of the assistant and the video annotated FOG events below. Middle: The freeze index calculated from the power in the freeze band divided by the power in the locomotor band. Bottom: Automatically as FOG detected parts (signal $=1$ ) together with the annotation as in the top graph. In this example all three FOG events were identified as such, with little delay. There are four 'false alarms': the beginning and the end of voluntary standing as well as two transient walking parts were detected as FOG.

using a 'freeze' threshold. FI values above this threshold are identified as FOG.

We developed an online FOG detection algorithm based on the principle described by Moore with emphasis on low latency. The Context Recognition Network (CRN) Toolbox [18] was used for the data processing. Only the shank sensor data has been used for the online FOG detection. Sensor data coming from the acceleration sensor with $64 \mathrm{~Hz}$ are windowed by a 256 sample window $(4 \mathrm{sec})$. The windowing is done in steps of 32 samples $(0.5 \mathrm{sec})$. On each window block the first 32 frequency components $(0-8 \mathrm{~Hz})$ of the power spectrum of a 256 point fast Fourier transformation (FFT) are calculated. The FFT calculation is the main computational part of the algorithm.

The energy in the low frequency part between 0.5 and $3 \mathrm{~Hz}$ is summed up as well as the energy in the higher frequency part between 3 and $8 \mathrm{~Hz}$. Furthermore the complete energy between 0.5 and $8 \mathrm{~Hz}$ is calculated by the addition of both parts. If the power content of the signal between $0.5-8 \mathrm{~Hz}$ is above the power-threshold PowerTH, the FI is calculated by dividing the energy in the freeze band by the energy in the locomotor band. FI is set to zero for signal parts with a power content below PowerTH, which are the standing parts. FOG is detected whenever the FI exceeds the 'freeze' threshold FreezeTH. The reduced window size and the second threshold (PowerTH) are necessary modifications of the algorithm described by Moore to make it online applicable. A signal extract together with the online calculated FI and the FOG detection signal is depicted in figure 2 .

\section{PROOF OF CONCEPT STUDY}

\section{A. Participants}

For our study idiopathic PD patients with a history of FOG, able to walk un-assisted in $O F F$ period were recruited by the specialists at the Movement Disorders Unit, Department of Neurology at the Tel Aviv Sourasky Medical Center (TAMSC). Patients unable to walk un-assisted in $O F F$ period have been

\begin{tabular}{cccccc}
$\begin{array}{c}\text { Subject } \\
\text { ID }\end{array}$ & Gender & $\begin{array}{c}\text { Age } \\
\text { [years] }\end{array}$ & $\begin{array}{c}\text { Disease duration } \\
\text { [years] }\end{array}$ & $\begin{array}{c}\text { H\&Y } \\
\text { in ON }\end{array}$ & Tested in \\
\hline 01 & M & 66 & 16 & 3 & OFF \\
02 & M & 67 & 7 & 2 & ON \\
03 & M & 59 & 30 & 2.5 & OFF \\
04 & M & 62 & 3 & 3 & OFF \\
05 & M & 75 & 6 & 2 & OFF \\
06 & F & 63 & 22 & 2 & OFF \\
07 & M & 66 & 2 & 2.5 & OFF \\
08 & F & 68 & 18 & 4 & ON \\
09 & M & 73 & 9 & 2 & OFF \\
10 & F & 65 & 24 & 3 & OFF \\
\hline Mean & & 66.4 & 13.7 & 2.6 & \\
\pm STD & & \pm 4.8 & \pm 9.67 & \pm 0.65 &
\end{tabular}

TABLE I

DETAILED PATIENTS CHARACTERISTICS OF AGE, DISEASE DURATION AND H\&Y RATING.

excluded as well as patients with severe vision or hearing loss, dementia or presence of other neurological/orthopedic diseases which might affect gait. The study was approved by the local Human Subjects Review Committee, and was performed in accordance with the ethical standards of the 1964 Declaration of Helsinki. Each subject signed an informed consent prior to entering the study. Ten PD patients diagnosed with idiopathic PD (7 males, 3 females; $66.5 \pm 4.8$ years; $2.7 \pm 0.6 \mathrm{H} \& \mathrm{Y}$ score in $O N$ ) have taken part at this study. Eight patients were examined in the $O F F$ state (more than $12 h$ after their last medication) and 2 patients, who regularly experience $O N$ FOG, were examined in the $O N$ state. The diversity of the participating patients covered a wide spectrum of PD patients. It included PD patients difficult to differentiate from healthy people by their gait performance when they do not have an FOG event, as well as one PD patient not able to walk distances $>15 m$, often using a wheel chair in normal life. The detailed patients characteristics are listed in table I.

\section{B. Protocol}

Subjects were asked to come to the TASMC for an experimental session that took about $1 \mathrm{~h}$. Patients arrived at 8:00 and 9:30am (two recordings per day) at the TASMC without having taken their usual morning PD medications, i.e., during the $O F F$ state (except the two, who regularly experience FOG in $O N$ state). First, patients were instructed on the experiment and explained how they may take advantage of the auditory cue in case of freezing.

The study protocol had two sessions and has been designed to represent normal daily walking (straight line, turning, and moving around rooms). Each sessions had three walking tasks, which consisted out of: (a) straight baseline walking in the 
lab hallway, including several 180 degrees turns; (b) random walking in a reception hall space that included a series of initiated stops and several 360 degrees turns - the examiner spontaneously instructed the subject to turn in different directions, at least six turns, three to each direction; (c) walking related to activities of daily living (ADL) - the ADL task included entering and exiting rooms, going to the lab kitchen, getting something to drink and going back with the cup of water to the starting room.

Figure 3 depicts a sketch of the path taken by the patients for each walking task. Each single walking task has been performed for about 5-10 min. Patients walked without assistance at their own natural pace, but with a therapist close by for safety reasons.

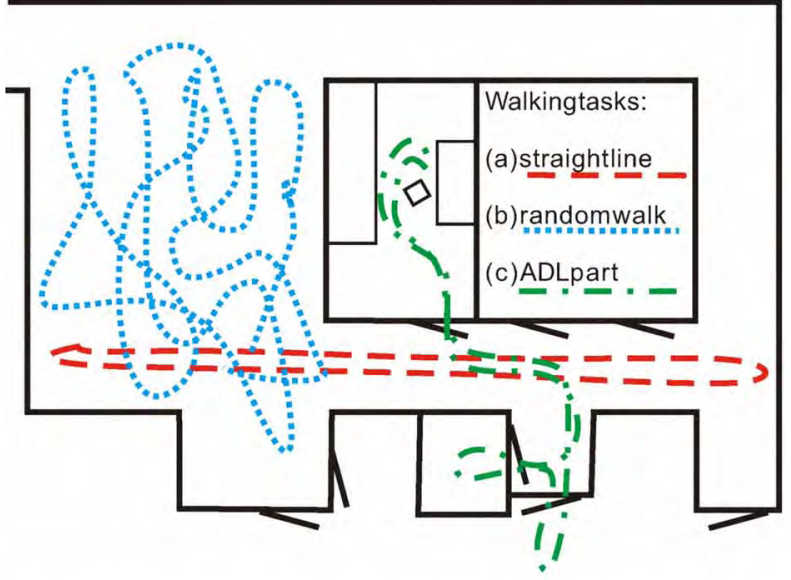

Fig. 3. Thumbnail sketch of the path taken by the subjects during the study.

During the first session the device recorded all the necessary data and performed online the FOG detection, however the metronome was deactivated. The second session is the repetition of the first session with the feedback system (metronome) activated.

At the end of the study patients returned to the examining room, took their medication, had a debriefing with the therapist and filled out a standardized self-report of patient satisfaction and a questionnaire to qualify the system's operation.

\section{Annotation of Ground Truth}

Figure 4 shows a snapshot of the study with two physiotherapist and two assistants, who were running and observing the study. Each patient was watched closely by an assistant who real-time annotated the patient's current activity (standing, walking, turning and freezing). For this purpose a general Laptop was used. The assistant could annotate all actions by pressing a corresponding key on the keyboard.

In addition, all walking trials were recorded on a digital video camera. Synchronization of leg movement data with the video recordings was achieved by recording three synchronization steps (resulting in characteristic and easy deterministic peaks in the acceleration signal) at the beginning of each recording session. In a post-hoc process, professional

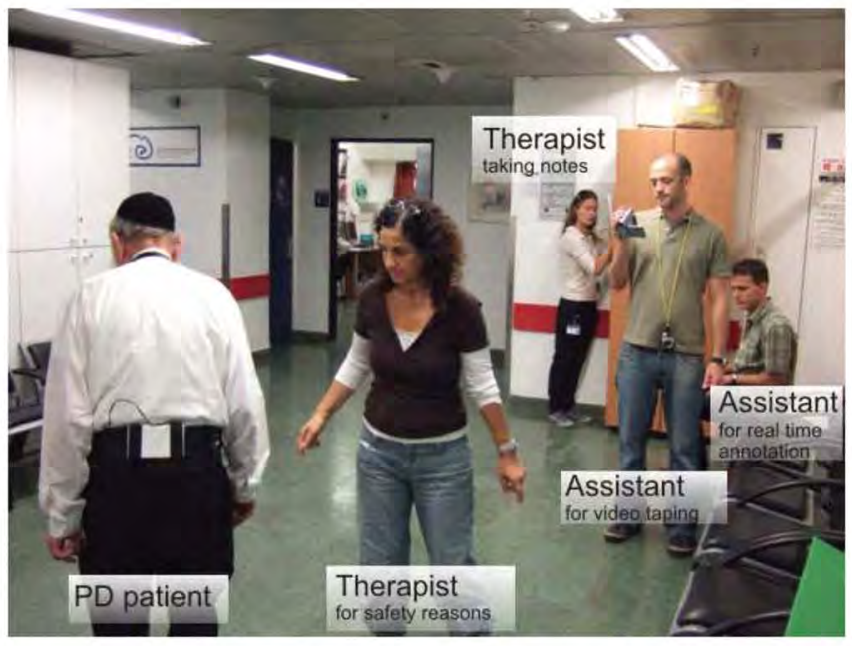

Fig. 4. Snapshot of the study, depicting one PD patient, the therapist (near the subject for safety reasons) and the research assistants (more remotely from the patient) who were documenting the trials

physiotherapists analyzed the video recordings to identify the FOG events and determine the exact start times, durations and end times.

\section{Subjective evaluation of the study}

We asked the patients to fill out a questionnaire for a subjective evaluation of the study The visual analogue scale (VAS) and the Clinical Global Impression Change scale (CGIC) were used for the questions where the patients have to give a selfassessment.

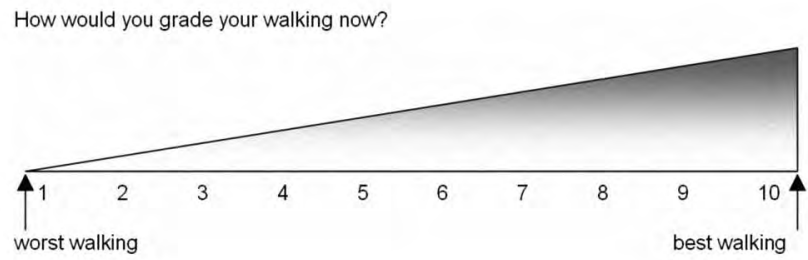

Fig. 5. Visual Analog Scale (VAS) e.g. about walking performance at the moment.

The VAS is a visual analogue scale with two anchor points, one at each extreme, as depicted in figure 5. Respondents specify their level of agreement to a statement by indicating a position on the VAS along a continuous line between the two end-points. The sensitivity and reproducibility of the results of an analogue scale are broadly similar to discrete scales, although the VAS may outperform the discrete scales in some cases [19].

The CGIC scale is a seven point scale that assesses how much the patient's performance or illness has improved or worsened relative to a baseline state at the beginning of the intervention, as used in [20]. For instance, figure 6 shows the scale used to self-report the change in duration of freezing episodes due to our system: $+3=$ very much longer; +2 , much 
longer; +1 , minimally longer; 0 , no change; -1 , minimally shorter; -2 , much shorter; or -3 , very much shorter.

Did you have longer/shorter freezing episodes with the device?

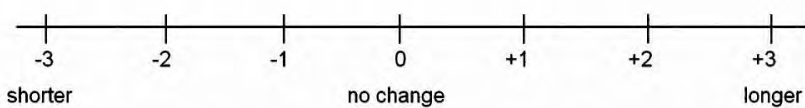

Fig. 6. Clinical Global Impression Change (CGIC) for questions concerning e.g. relative amount of FOG events, relative length of FOG events or preferred relative occurrence of rhythmic assistance compared to experience in the study.

Before starting the study, we had one pre-study question, where the patients had to grade their walking performance before the study. In the post-study questionnaire, after the study, we asked again the question about their walking performance, now of how they grade their walking during the study. Furthermore, we had questions using the VAS concerning the comfort and the disturbance of the belt with the computing device and the sensors at the leg, with anchor points did disturb 'not at all' / 'very much' while walking. Finally we used the VAS to asked the patients if they think such a system could increase their Quality of Life (QoL) on a scale from 'not at all' to 'very much'.

For questions concerning relative changes the CGIC scale was used. Questions addressed:

- The relative occurrence of freezing events with the device compared to before (less / no change / more).

- The relative length of freezing events with the device compared to before (shorter / no change / longer).

- The preferred occurrence of the metronome assistance relative to the experience in the study (less often / no change / more often).

In all cases there was the opportunity to give additional comments to the ratings. Within the questionnaire we also asked the patients if they would be willing to wear the system a whole day and if they would like to change something (e.g. place of attachment, kind of feedback, ... ).

The physiotherapists answered a complementary questionnaire at the end of the complete study to get a second evaluation from another perspective.

\section{RESUlTS AND Discussion}

\section{A. Objective results}

Eight out of the ten patients exhibited FOG during the study, two patients did not have any FOG event. The walking distance and number of turns depended on the patients' gait speed. One patient could not perform the ADL part.

Overall, $8 \mathrm{~h} 20 \mathrm{~min}$ of data have been recorded. 237 FOG events (range 0-66 per patient; mean 23.7 [S.D. 20.7]) have been identified from the video recordings by the physiotherapists. The detailed distribution of number of FOG per patient is given in figure 7.

The length of the FOG events ranged from $0.5 \mathrm{sec}$ to $40.5 \mathrm{sec}$ (mean $7.3 \mathrm{sec}$ [S.D. $6.7 \mathrm{sec}$ ]). $50 \%$ of the FOG

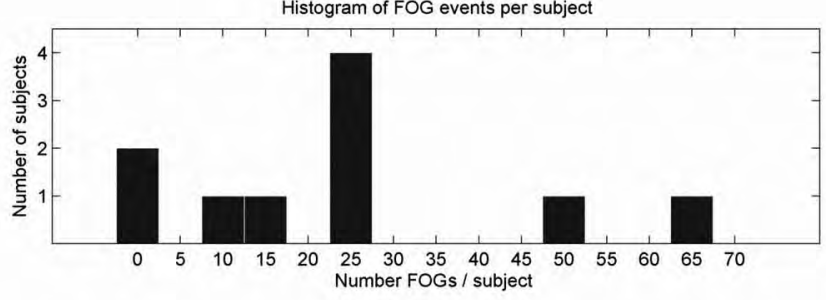

Fig. 7. Distribution of number of FOG events per patient.

episodes lasted less than $5.4 \mathrm{sec}$, and the majority of $93.2 \%$ of FOG events had a duration less than 20 sec. The detailed distribution of number of FOG events within $1 \mathrm{sec}$ bins is given in figure 8 .

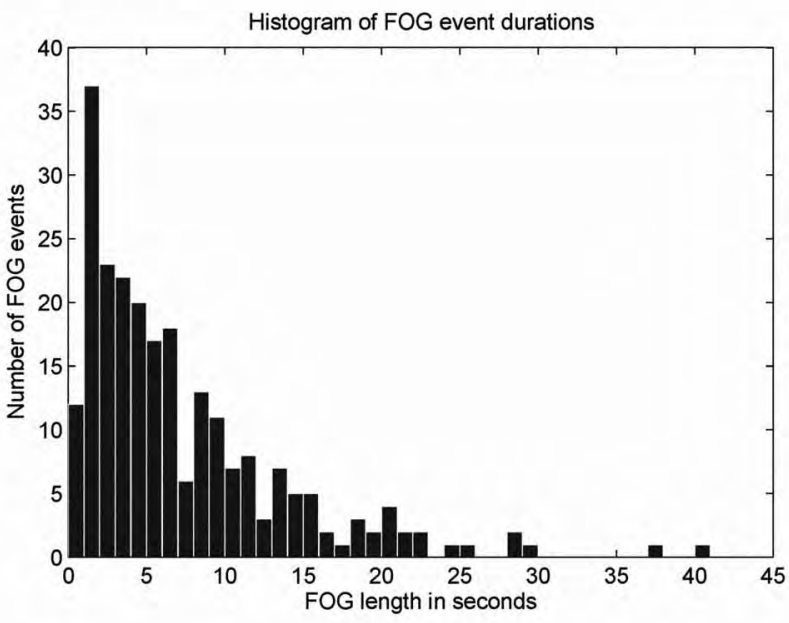

Fig. 8. Distribution of duration of FOG events.

No technical malfunctions occurred during the recordings. With no exceptions, the device initiated auditory cueing whenever a FOG episode was detected, and turned off when the system identified the termination of the episode. $96.2 \%$ of the identified FOG episodes $(n=237)$ were detected online by the wearable device. The frame based sensitivity and specificity of the online detected FOG were $73.1 \%$ and $81.6 \%$, respectively. The evaluation is based on $0.5 \mathrm{sec}$ frames.

Figure 9 depicts the detection accuracy of the device. For each patient sensitivity value (abscissa) and specificity value (ordinate) is plotted. It can be seen that the system did not work equally well for all patients. Worst results in terms of specificity performance was obtained for patient 01 . Only $39.7 \%$ specificity was achieved (with $99.1 \%$ sensitivity). Worst results in terms of sensitivity was obtained for patient 08 . Only a sensitivity of $34.1 \%$ (with specificity of $88.9 \%$ ) was achieved.

These large variations result from the different walking styles of the patients. For example, patient 01 suffered from foot drop while walking. Most of the time the system was not able to distinguish between walking periods and very short freezing events using the global algorithm parameter settings. Results of $95.9 \%$ sensitivtiy with $92.7 \%$ specificity could be 


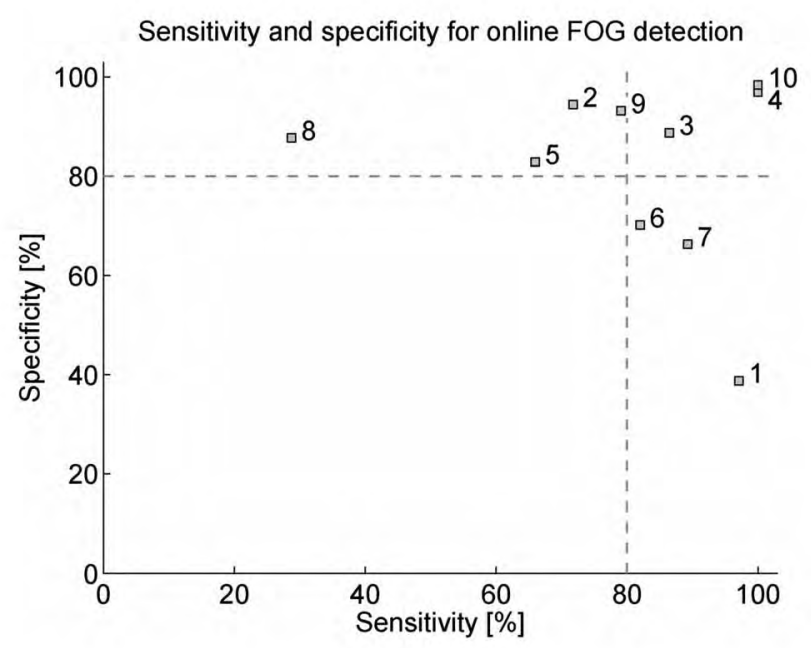

Fig. 9. Sensitivity and specificity distribution for the online detection accuracy. Numbers correspond to the patients ID.

achieved for patient 01 by adapting the two threshold parameters of the algorithm to a FreezeTH of 3.0 (= FreezeTH study $_{\text {. }}$. 1.5). For patient 08 , best results of $76.1 \%$ sensitivity and $78.1 \%$ specificity are achiveable with the parameter setting of FreezeTH equals $0.5\left(=\right.$ FreezeTH $\left._{s t u d y} \cdot 0.25\right)$. A detailed performance characterization based on the parameter setting is presented and discussed in Bächlin et al. [21]. If such a system becomes a 'consumer appliance', provision for an initial calibration would be beneficial.

\section{B. Subjective questionnaire results}

Each patient had about $30 \mathrm{~min}$ to use the system. Therefore, the questionnaires can only provide first user impressions. The main objective of this study was to evaluate the subjective perception of the context-aware assistance. Additionally we asked the patients on the optimal form factor as further input for future developments.

Unequivocal patients' responses suggest that the system was unobtrusive and did not interfere with their locomotion. The majority of patients ( $\mathrm{n}=9)$ chose the 'not at all disturb' option, when responding to whether the computing device and the sensors (as physical objects with volume and weight) obstructed their gait. Only one rated slightly above 'not at all disturbing' for the sensor at the leg and mentioned, that he was feeling the elastic strap (sensor) at his leg. He felt like under observation and this called his attention to concentrate on his walking performance.

All ten patients expressed their willingness to wear the sensors during every day life. None had any privacy concerns of collecting their gait data. One patient said, he would wear it even for months if necessary for further research. This are positive preliminary results as a follow-up study with the system worn over a longer time is required for a clear assessment of the effectiveness and wearability in daily life.

Although the computing system is still quite big, there was only one comment that it should be smaller or even should work without. The belt seems to be the most preferable place to carry such a device. Nobody wanted to wear it somewhere else. Also the sensor attachment to the ankle is an acceptable place for all patients, as long as the sensor can be worn below the pants. However, the sensor position just above the knee should be avoided.

Two of the physiotherapist thought that the wearable system is already suitable for day long at home recordings with minor drawbacks. The other two have seen it less suitable, especially because the computing system is not permanently attached to the belt and too large, and there is no professional housing for the sensors.

We used headphones placed around the patients neck as loudspeaker to know directly during the study when the auditive feedback is working and also to hear it on the video recordings. This may not be the optimal configuration for everyday usage. Also the patients commented most on this issue. Some of the responses we received from the patients suggested that earphones should be less visible. In particular, they noted it is very desirable that people in the surroundings will not notice they are wearing such system, either by seeing it or by hearing the ticking sound. One patient expressed his desire for a visual feedback. Such an visual feedback could be lines projected on the floor whenever he has an FOG event.

The responses about the effectiveness of the system were more heterogeneous. Five out of the eight patients, who experienced FOG during the study, thought they had less freezing events with the device. The remaining three could not see any change. Also five patients had the impression their
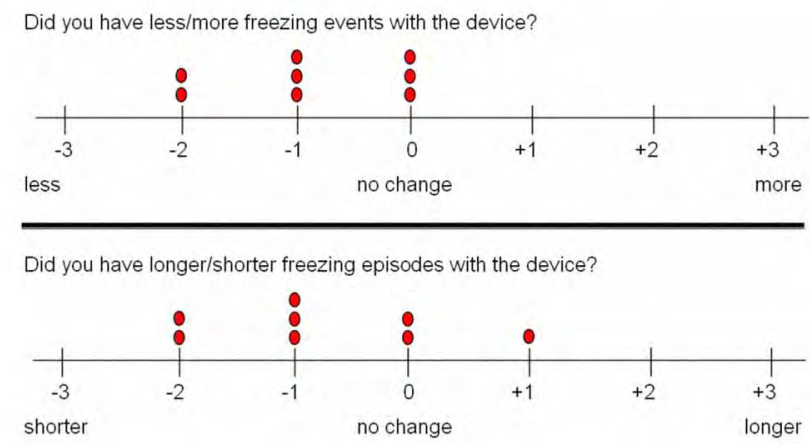

Fig. 10. CGIC questionnaire results concerning relative amount of FOG events and relative length of FOG events during the study

freezing episodes have been shorter with the device. Only one thought his episodes have been longer as normal, and two could not determine any change. The answers are depicted in figure 10 .

The observation of having less freezing events correlates for four patients with the observation of having shorter freezing episodes $(02,03,09$ : less + shorter freezing events, 06: much less + much shorter freezing events). Patient 07 could not see that he had less or more freezing events, however, the length of freezing was much shorter. Patient 08 had the feeling that she experienced less freezing events, however these have been longer as usually. Two had not experienced any change in 
number of FOG occurrences, neither in their length.

Overall, these self-assessment indicate that the patients benefit from the system - or at least had the impression to benefit from it. Also the physiotherapists rated the influence of the automatic identification of the FOG events and the auditive feedback as beneficial, at least for those with severe freeze. Observations by the physiotherapists have shown that the patients used the cueing mainly in long turning freeze which are longer than $3 \mathrm{sec}$, and in tight quarters (ADL part) after they were stuck. The rhythmic sound helped them to take small steps to come out of the freezing. One physiotherapist differentiated between patients, that are usually more or less stable during the freeze and patients, that almost fall due to FOGs. Patients, that usually are more or less stable during the freeze will use the cue more while freezing to get out again. Patients, that almost fall due to FOGs will try to use the cues to prevent falling. Therefore, the cueing should already assist these people while they are walking, as Rhythmic Auditory Stimulation (RAS), so that they can use the rhythm to improve their walking abilities.

The responses regarding the occurrence of the rhythmic assistance by the metronome sound are depicted in figure 11 and cover the whole spectrum from 'should appear more often' to 'should appear less often'.

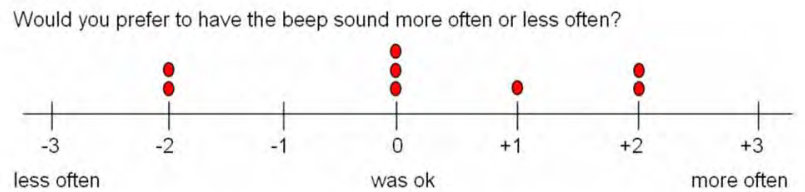

Fig. 11. CGIC questionnaire results concerning preferred relative occurrence of rhythmic assistance compared to experience in the study.

Two patients clearly expressed their preference to hear the metronome less often. Looking at the performance of the system showed that it was too sensitive for these patients. A too sensitive system results in too many false alarms, which is annoying for the patients. It also shows that a continuous cueing is not acceptable for these people.

On the other hand, three patients expressed their preference to have the metronome sound more, or even much more often. Also these answers are quite coherent with the observation of the system performance during the study. At least in two cases the sensitivity was relatively low, resulting in missed FOG events and therefore the patients did not always get the auditory assistance when they experienced an FOG event. For the third patient we could not see such a clear indication (low sensitivity). Personal preferences are the most probable reason. However, their expression to get the auditive assistance more often compared to what they have experienced during the study indicates that they seem to have benefited from it.

For three patients the performance was just right. One mentioned that the beep sound and rhythm always was the same and he got used to it. He suggested to bring in some variations, because he thought, then it would help him even more. Similarly this was also a comment by the physiotherapists: the device should adjust the tempo according the the walking speed of the patient.

The question about the possibility of such a system to increase their QoL was answered relatively positively by six patients between 5-9 (see figure 12). For the remaining four the trial was too short, and they can not really judge. Also three physiotherapists rated the possibility of an automatic external cueing device to increase the QoL relatively high between 6-9. One physiotherapist was more skeptical and rated a 3 , because

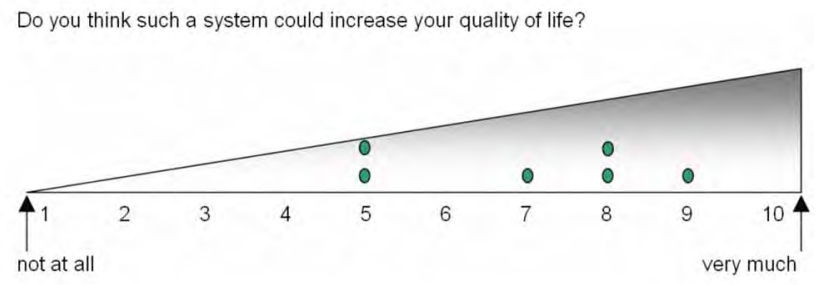

Fig. 12. Visual Analog Scale (VAS) about possible 'Quality of Life' improvements

she thinks it depends very much on the patient. However, she thinks that for PD patients which have long FOG events and are much aware, attentive and capable to adapt to the rhythm, automatic cueing will improve their QoL.

\section{CONCLUSION}

To our knowledge this is the first time that FOG has been automatically detected online by a wearable device. The system detected the FOG events with a sensitivity of $73.1 \%$ and a specificity of $81.6 \%$. The evaluation responses obtained from the patients were rather positive, despite the fact that the first prototype is relatively bulky. This fact is promising for future utilization of this concept. Some patients expressed their motivation to wear it even for several weeks. Also the answers regarding the influence and the effect of the automatic cueing was promising. At least half of the patients have seen a positive effect. The demand for more frequent auditive feedback of the patients, for whom the system had a low sensitivity shows, that these people had the feeling the sound helps them. On the other hand, the demand for less auditive feedback of the patients, for whom the system had a low specificity discloses, that a continuous cueing is annoying. These results demonstrate the need for a context aware system and motivate further studies.

One limitation of this study is the short time the patients experienced the system. Another limitation is the laboratory setup, where the system was tested, instead of a natural daily living surrounding. Despite the fact that all the patients reported on a history of FOG, two of them did not experience any FOG during the experiments. It is known that a laboratory setting can cause changes in attention and stress levels, which may result in reduction of FOG episodes. In addition, the controlled environment of the study and the physiotherapist nearby may have reduced the likelihood of FOG in the two patients, who did not experience any FOG event during the 
study. Both patients reported lots of freezing at home and could not explain why they did not have any FOG during the study. They expressed the motivation to test our device during their natural daily activities.

Further investigations are necessary to analyze and prove the real-world performance. Remaining open questions are: (i) How useful will the patients find the system when using it for 2-3 hours for several days; (ii) During constant use, can the patients learn to rely on the system the way they rely on other walking aids? (iii) Can they even get used to it so much, that the cueing sound is not disturbing?

Although the system was already well accepted by the patients, there are further possibilities for technical improvements. The whole miniaturized computing system with all its functionality can be integrated in the buckle of a belt [22]. A more specialized system, especially designed for this task can even be miniaturized to a size of a button with the FOG algorithms included in the sensor node itself. Roggen et al. have shown that even complex calculations such as FFT, which is used in the online detection of FOG, can be processed with low power consumption on a device of the size of a button [23]. Such a system could be entirely integrated into (or attached to) normal shoes of the patient, and only the trigger for the external cueing signal is transmitted to the feedback device. The external cueing signal could be given by a hearing aid like device or even the hearing aid itself.

\section{ACKNOWLEDGMENT}

This paper describes work carried out in the context of the DAPHNet project, 'Dynamic Analysis of Physiological Networks'. DAPHNet is an Future and Emerging Technologies (FET) project supported by the European 6th Framework Program, Grant No. 018474-2. We thank the patients for their participation, time and effort, and Ms. Inbal Meidan, Ms Talia Herman, Ms Marina Brozgol and Mr. Eliya Shaviv for their invaluable assistance.

\section{REFERENCES}

[1] H. Braak, E. Ghebremedhin, U. Rüb, H. Bratzke, and K. D. Tredici, "Stages in the development of parkinson's disease-related pathology," Cell and Tissue Research, vol. 318, no. 1, pp. 121-134, October 2004.

[2] J. Jankovic, "Parkinson's disease: clinical features and diagnosis," $J$ Neurol Neurosurg Psychiatry, vol. 79, no. 4, pp. 368-376, 2008.

[3] M. M. Hoehn and M. D. Yahr, "Parkinsonism: onset, progression and mortality," Neurology, vol. 17, no. 5, pp. 427-42, May 1967.

[4] S. Fahn, "The freezing phenomenon in parkinsonism," Adv Neurol., vol. 67, pp. 53-63, 1995.

[5] J. D. Schaafsma, Y. Balash, T. Gurevich, A. L. Bartels, J. M. Hausdorff, and N. Giladi, "Characterization of freezing of gait subtypes and the response of each to levodopa in parkinson's disease," European Journal of Neurology, vol. 10, no. 4, pp. 391-398, 2003.

[6] M. Macht, Y. Kaussner, J. C. Mller, K. Stiasny-Kolster, K. M. Eggert, H.P. Krger, and H. Ellgring, "Predictors of freezing in parkinson's disease: A survey of 6,620 patients," Movement Disorders, vol. 22, no. 7, pp. 953-956, 2007.

[7] B. R. Bloem, J. M. Hausdorff, J. E. Visser, and N. Giladi, "Falls and freezing of gait in parkinsons disease: A review of two interconnected, episodic phenomena," Movement Disorders, vol. 19, no. 8, pp. 871-884, 2008.
[8] A. G. de Boer, W. Wijker, J. D. Speelman, and J. C. de Haes, "Quality of life in patients with parkinson's disease: development of a questionnaire," Journal of Neurology, Neurosurgery and Psychiatry, vol. 61 , no. 1 , pp. 70-74, July 1996 .

[9] N. Giladi, M. P. McDermott, S. Fahn, S. Przedborski, J. Jankovic, M. Stern, and C. Tanner, "Freezing of gait in PD: Prospective assessment in the DATATOP cohort," Neurology, vol. 56, no. 12, pp. 1712-1721, 2001.

[10] M. H. Thaut, G. C. McIntosh, R. R. Rice, R. A. Miller, J. Rathbun, and J. M. Brault, "Rhythmic auditory stimulation in gait training for parkinsons disease patients," Movement Disorders, vol. 11, no. 2, pp. 193-200, 1996.

[11] E. van Wegen, C. de Goede, I. Lim, M. Rietberg, A. Nieuwboer, A. Willems, D. Jones, L. Rochester, V. Hetherington, H. Berendse, J. Zijlmans, E. Wolters, and G. Kwakkel, "The effect of rhythmic somatosensory cueing on gait in patients with parkinson's disease," Journal of the Neurological Sciences, vol. 248, no. 1-2, pp. 210-214, 2006.

[12] I. Lim, E. van Wegen, C. de Goede, M. Deutekom, A. Nieuwboer, A. Willems, D. Jones, L. Rochester, and G. Kwakkel, "Effects of external rhythmical cueing on gait in patients with parkinson's disease: a systematic review," Clinical Rehabilitation, vol. 19, no. 7, pp. 695-713, 2005.

[13] A. Nieuwboer, G. Kwakkel, L. Rochester, D. Jones, E. van Wegen, A. M. Willems, F. Chavret, V. Hetherington, K. Baker, and I. Lim, "Cueing training in the home improves gait-related mobility in Parkinson's disease: the RESCUE trial," Journal of Neurology, Neurosurgery, and Psychiatry, vol. 78, no. 2, pp. 134-140, 2007.

[14] M. Bächlin, D. Roggen, and G. Tröster, "Context-aware platform for long-term life style management and medical signal analysis," in In Proceeding of the 2nd SENSATION International Conference, Chania, Greece, June 2007.

[15] J. D. Schaafsma, N. Giladi, Y. Balash, A. L. Bartels, T. Gurevich, and J. M. Hausdorff, "Gait dynamics in parkinson's disease: relationship to parkinsonian features, falls and response to levodopa," Journal of the Neurological Sciences, vol. 212, no. 1-2, pp. 47-53, 2003.

[16] J. M. Hausdorff, Y. Balash, and N. Giladi, "Time series analysis of leg movements during freezing of gait in parkinson's disease: akinesia, rhyme or reason?" Physica A: Statistical Mechanics and its Applications, vol. 321 , no. 3-4, pp. 565-570, 2003.

[17] S. T. Moore, H. G. MacDougall, and W. G. Ondo, "Ambulatory monitoring of freezing of gait in parkinson's disease," Journal of Neuroscience Methods, vol. 167, no. 2, pp. 340-348, 2008.

[18] D. Bannach, K. Kunze, P. Lukowicz, and O. Amft, "Distributed modular toolbox for multi-modal context recognition," in ARCS 2006: Proceedings of the 19th International Conference on Architecture of Computing Systems., March 2006, pp. 99-113.

[19] S. Grant, T. Aitchison, E. Henderson, J. Christie, S. Zare, J. McMurray, and H. Dargie, "A Comparison of the Reproducibility and the Sensitivity to Change of Visual Analogue Scales, Borg Scales, and Likert Scales in Normal Subjects During Submaximal Exercise," Chest, vol. 116, no. 5, pp. 1208-1217, 1999.

[20] R. C. Shelton, M. B. Keller, A. Gelenberg, D. L. Dunner, R. Hirschfeld, M. E. Thase, J. Russell, R. B. Lydiard, P. Crits-Christoph, R. Gallop, L. Todd, D. Hellerstein, P. Goodnick, G. Keitner, S. M. Stahl, and U. Halbreich, "Effectiveness of St John's Wort in Major Depression: A Randomized Controlled Trial," Journal of the American Medical Association, vol. 285, no. 15, pp. 1978-1986, 2001.

[21] M. Bächlin, D. Roggen, M. Plotnik, J. Hausdorff, and G. Tröster, "Online detection of freezing of gait in parkinson's disease patients: A performance characterization," in Proceedings of the 4th Int. Conf. on Body Area Networks, 2009.

[22] O. Amft, M. Lauffer, S. Ossevoort, F. Macaluso, P. Lukowicz, and G. Tröster, "Design of the qbic wearable computing platform," in ASAP '04: Proceedings of the Application-Specific Systems, Architectures and Processors, 15th IEEE International Conference on (ASAP'04). Washington, DC, USA: IEEE Computer Society, 2004, pp. 398-410.

[23] D. Roggen, N. B. Bharatula, M. Stäger, P. Lukowicz, and G. Tröster, "From sensors to miniature networked sensorbuttons," in In Proc. of the 3rd Int. Conf. on Networked Sensing Systems (INSSO6), 2006, pp. 119-122. 